\title{
TA Spectrum
}

\author{
Dmitri Ivanov $^{1 *}$, for the Telescope Array collaboration \\ ${ }^{1}$ University of Utah, High Energy Astrophysics Institute, Salt Lake City, Utah, USA
}

\begin{abstract}
The Telescope Array (TA) experiment is measuring cosmic rays of energies from PeV to $100 \mathrm{EeV}$ and higher in the Northern hemisphere. TA has two parts: main TA and the TA low energy extension (TALE). Main TA is a hybrid detector that consists of 507 plastic scintillation counters on a 1200m - spaced square grid that are overlooked by three fluorescence detector stations. TALE is also a hybrid detector that consists of additional fluorescence telescopes arranged to view higher elevations and an infill array of 103 plastic scintillation counters. In this work, we describe the combined TA surface detector (SD) and TALE fluorescence detector spectrum, check the calculation of the TA SD spectrum at the highest energies using an alternative, Constant Intensity Cut, method and discuss the declination dependence of the TA SD spectrum at the highest energies. Details of the TALE spectrum calculation have been presented in a separate work at this conference.
\end{abstract}

\section{Introduction}

Energy spectrum is an important tool in cosmic ray physics. Spectral features including the knee at $10^{15.5} \mathrm{eV}$, the ankle near $\sim 10^{18.5} \mathrm{eV}$, the cutoff near $\sim 10^{19.5} \mathrm{eV}$, and, recently measured, the second knee at $\sim 10^{17.0} \mathrm{eV}$ and the low energy ankle at $\sim 10^{16.2} \mathrm{eV}[3]$, as well as the corresponding spectral indices, provide information about the nature of the cosmic ray sources and the effects of the cosmic ray propagation. The Telescope Array (TA) is a modern cosmic ray detector, which is sensitive over a wide range of energies and which sees these features.

In this paper, we discuss the combined TA energy spectrum measurement, ranging from a couple of $\mathrm{PeV}$ to hundreds of $\mathrm{EeV}$ and higher, and focus on the recent developments of the TA surface detector (SD) spectrum analysis at the highest energies: a declination dependence of the TA SD spectrum above $10^{19} \mathrm{eV}$, a discrepancy in the spectrum measurement between the TA and Auger experiments above $10^{19} \mathrm{eV}$, and an important check of the TA SD spectrum calculation using the Constant Intensity Cut [1] analysis, which is an alternative model-independent reconstruction technique for TA. Current details of the TALE spectrum measurement and interpretation can be found in [2],[3], and [4].

\section{TA Data}

TA is located in the desert of Millard County, UT, USA at an altitude of $\sim 1400 \mathrm{~m}$ above sea level, which corresponds to $\sim 880 \mathrm{~g} / \mathrm{cm}^{2}$ vertical mass overburden. TA is designed to measure cosmic rays using the ground array and air fluorescence detection techniques. The main TA, which has

*e-mail: dmiivanov@gmail.com been in operation since 2008, consists of a surface detector array of 507 plastic scintillation counters deployed on a square grid of $1200 \mathrm{~m}$ spacing [5], which is overlooked by 3 fluorescence detector (FD) stations [6,7]. Each TA SD counter uses 2 layers of $3 \mathrm{~m}^{2} \times 1.2 \mathrm{~cm}$ plastic scintillator.

The TA low energy extension (TALE) consists of 10 additional fluorescence telescopes at the Northern TA fluorescence detector site, called the TA Middle Drum (MD), and an infill array of 103 counters of 400 and $600 \mathrm{~m}$ spacing in front of the TA MD site. The TALE fluorescence telescopes extend the TA FD field of view from the originally designed range of 3 to $33^{\circ}$ in elevation to $57^{\circ}$ in elevation. This allows a reconstruction of lower energy cosmic rays in fluorescence and Čerenkov mode by the TA FD, thus extending the sensitivity of the TA experiment from the original design range of $1 \mathrm{EeV}$ and higher down to a couple of PeV [3]. The TALE FD has been taking data since 2014.

\section{Combined TA Spectrum}

Figure 1 shows the combined TA energy spectrum using black filled circles. The spectra of the KASCADE-Grande [8] and the Pierre Auger Observatory [9] have been superimposed using red squares and green triangles, respectively. The combined TA spectrum, shown at the UHECR 2018 conference, consists of the 9 year TA surface detector spectrum [10] and the recently published 22 month TALE monocular spectrum, [3]. After rescaling of the Auger energies by a constant factor of $+10.2 \%$, there is a visible agreement among the three experiments, for the energies below $10^{19.4} \mathrm{eV}$. A significant discrepancy between TA and Auger occurs at the highest energies. We investigate this discrepancy in two ways: first, following the recent efforts of the TA-Auger spectrum working group [11, 12], we restrict the TA SD spectrum measurement to the declination 


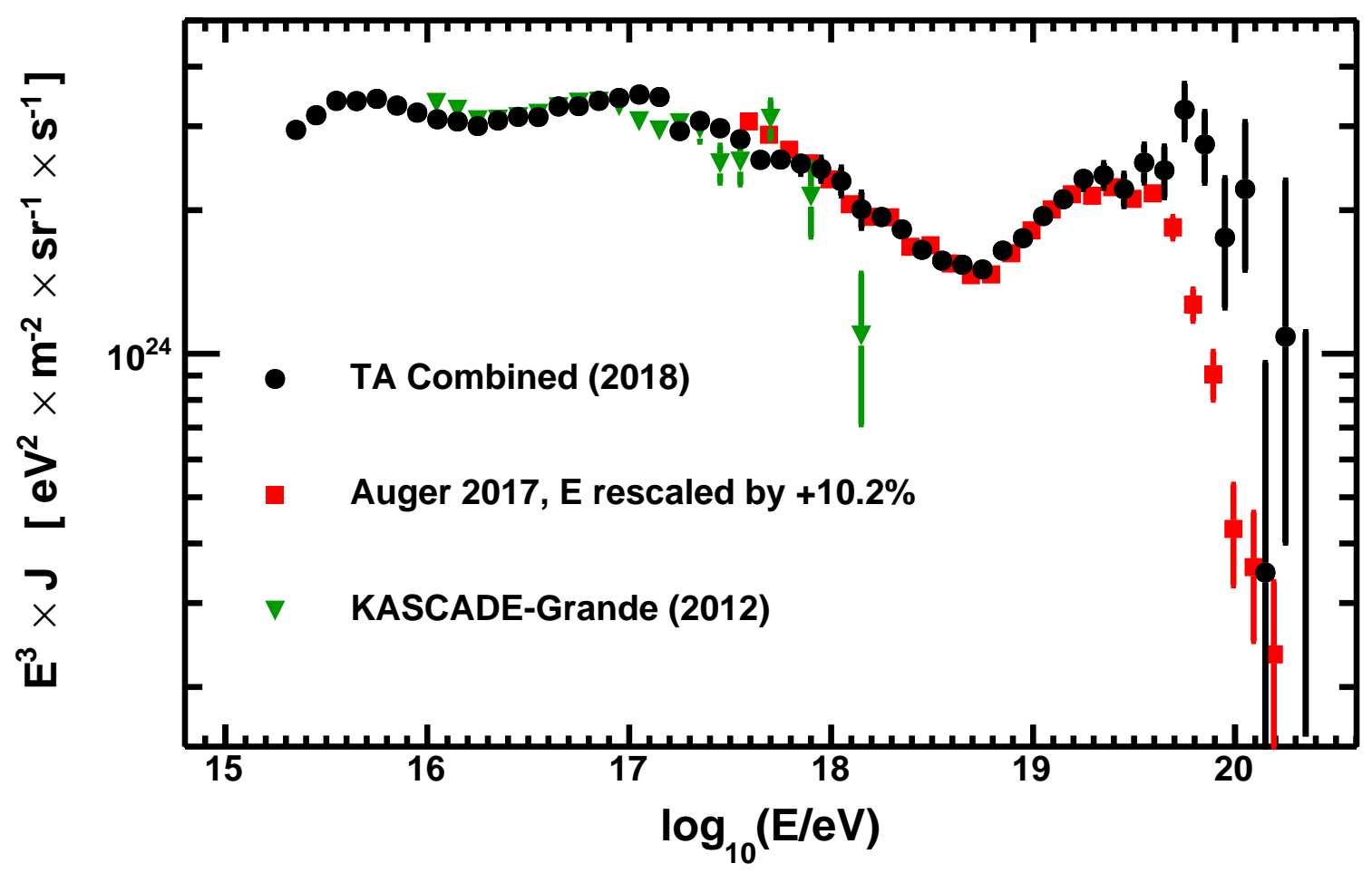

Figure 1. The combined TA spectrum (black points) presented at the UHECR-2018 conference: energy range from $10^{15.3}$ to $10^{18.3} \mathrm{eV}$ is covered by the TALE FD [3], while the TA SD measurement [10] starts at $10^{18.2} \mathrm{eV}$ and extends to $10^{20} \mathrm{eV}$ and higher. Superimposed are the Auger combined spectrum [9], with the Auger energies rescaled by $+10.2 \%$ (red squares), and the KASCADE-Grande spectrum [8] (green triangles).

band that is within the field of view of the Auger, and second, we check the TA SD spectrum calculation using the Constant Intensity Cut [1] energy reconstruction technique, implemented in a way similar to that of the Pierre Auger experiment.

\section{Declination Dependence of the TA SD Spectrum}

Figure 2 shows the TA SD spectrum calculated for the lower and higher declination bands, which correspond to $-15.7^{\circ}<\delta<24.8^{\circ}$ and $24.8^{\circ}<\delta<90^{\circ}$, respectively. In the lower declination band, $-15.7^{\circ}<\delta<24.8^{\circ}$, which is visible to the Auger experiment, the cutoff energy of the TA spectrum occurs at a significantly lower energy of $10^{19.59_{-0.07}^{+0.05}} \mathrm{eV}$, which is well within $1 \sigma$ of the Auger result, $10^{19.62 \pm 0.02} \mathrm{eV}[11,12]$. The global chance probability of this effect has been evaluated using the Monte Carlo to be $3.5 \sigma$.

\section{Check of the TA SD Spectrum}

\subsection{Standard Reconstruction}

As it can be seen in [14] and [15], the standard reconstruction of surface detector events in TA is done as follows. First, counter time and pulse height information are fitted to determine the arrival direction of the event. Then, a fit of the counter pulse height information into the AGASA lateral distribution function [16] is performed, to determine $\mathrm{S} 800$, which is the signal size $800 \mathrm{~m}$ from the shower axis. An initial energy estimate, $E_{\text {MODEL }}[S 800, \sec (\theta)$ ], in terms of the reconstructed $\mathrm{S} 800$ and zenith angle, has been derived from a detailed CORSIKA [17] Monte Carlo, that uses proton QGSJET II.3 [18] hadronic interaction model, executed with the optimal thinning approximation [19], and de-thinned [20] to restore the important information on the ground. The final energy has been rescaled to the TA fluorescence detector using well reconstructed hybrid events seen by the TA SD and the TA FD in common: $E=$ $1 / 1.27 \times E_{\text {MODEL }}[14]$. In other words, in the case of the proton QGSJET II.3 model, the model-derived SD energy is related to the energy given by the fluorescence detector via the calibration factor 1.27: $E_{\mathrm{MODEL}}=1.27 \times E_{\mathrm{FD}}$.

Although the QGSJET II.3 hadronic model is used in the TA SD analysis by default, we have also calculated the SD - FD calibration factors corresponding to other hadronic interaction models, in order to estimate the possible non-linearity effects on the SD energy arising from the use of the hadronic model. The left panel of Figure 3 shows that the SD - FD calibration factor generally varies among the different hadronic interaction models. However, as the right panel of Figure 3 shows, after constant factors are taken out, the non-linearity effect of the most 


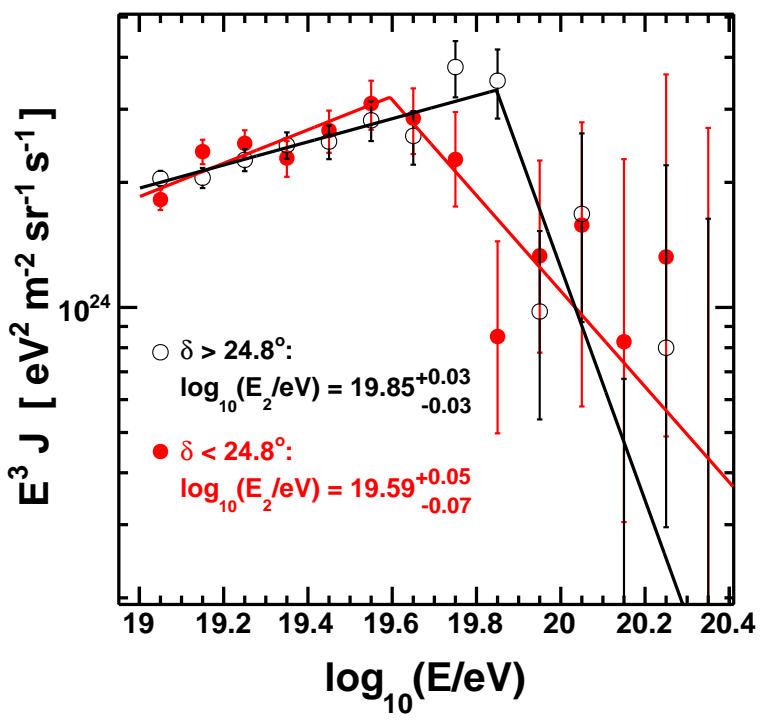

Figure 2. The TA SD energy spectrum in the two declination bands [13]. Filled red circles correspond to the spectrum in the lower declination band, $-15.7^{\circ}<\delta<24.8^{\circ}$, and open black circles represent the spectrum in the higher declination band, $24.8^{\circ}<\delta<90^{\circ}$. Solid lines show the broken power law fits that correspond to each declination band. The cutoff energies $\left(\log _{10}\left(E_{2} / \mathrm{eV}\right)\right)$ are $19.59_{-0.07}^{+0.05}$ for the lower and $19.85_{-0.03}^{+0.03}$ for the higher declination bands. The cutoff energies in the two declination bands are $4 \sigma$ different.

extreme case is well within $10 \%$ per decade of energy. Finally, we check the non-linearity of the SD energy by directly comparing the reconstructed SD energies (using proton QGSJET II.3 model) with the FD energies using hybrid events. As Figure 4 shows, there is no evidence of a non-linearity of the SD energy with respect to the FD.

\subsection{Constant Intensity Cut Method}

The $100 \%$ efficiency plateau of the TA SD occurs near $10^{19} \mathrm{eV}$. In the 9 years of operation, the TA SD has accumulated enough events to allow one to perform the Constant Intensity Cut (CIC) [1] analysis, which is a model-independent way of deriving the attenuation curve of the event S800 with respect to the event zenith angle. The left panel of Figure 5 shows the CIC curve derived from the TA SD data, and the right panel of Figure 5 shows the normalization of the energy estimator $S_{34}$ to the FD, from which we obtain the expression for the SD energy: $\log _{10}\left(E_{\mathrm{CIC}}^{\mathrm{SD}} / \mathrm{eV}\right)=([16.2 \pm 0.3]+$ $\left.\log _{10}\left[S_{34} /\left(\mathrm{VEM} \mathrm{m}^{-2}\right)\right]\right) /(0.93 \pm 0.02)$. Figure 6 shows the constant intensity cut procedure applied to the TA SD Monte Carlo. The data and Monte Carlo CIC attenuation curves agree, as the left panel of Figure 6 shows, and $S_{34}$ versus energy relationship obtained from the TA SD Monte Carlo agrees with that obtained from the data, as it can be seen by comparing the right panels of Figures 5 and 6. Next, Figure 7 shows the comparison of the TA SD energies reconstructed using the TA SD standard recon- struction method described in Section 5.1 and the reconstruction using the CIC method. The two methods, as it can be seen on the right panel of Figure 7, agree at a $\sim 3 \%$ level. Finally, as Figure 8 demonstrates by comparing the SD and FD energies of the hybrid events, the CIC method does not have an energy-dependent reconstruction bias.

\subsection{Spectrum Comparison}

Having described and validated the two independent methods of estimating the energy of the TA SD events, we can now compare the TA SD energy spectra, calculated by these two methods. Figure 9 shows the comparison of the TA SD spectra calculated using both methods in the lower (left panel) and the higher (right panel) declination bands. As it was expected from the energy comparisons of Figure 7 , the spectra in Figure 9 are in excellent agreement.

\section{Summary}

We have provided a combined TA spectrum measurement using the most recent results of the TA SD and TALE FD. The combined TA spectrum starts at $10^{15.3} \mathrm{eV}$ and extends to $10^{20.3} \mathrm{eV}$, covering 5 orders of magnitude in energy. Cosmic ray spectrum features the knee, the low energy an$k l e$, the second knee, the ankle, and the cutoff have all been observed in the TA data.

We have compared the combined TA spectrum to the spectra of the KASCADE-Grande and the Pierre Auger Observatory, and we have found a reasonable agreement over a wide range of energies, excluding the energies above $10^{19.4} \mathrm{eV}$, where the TA and Auger full sky spectra have a significant discrepancy. We have established that this TA and Auger discrepancy can be, in part, explained by the $3.5 \sigma$ (global significance) declination dependence of the TA SD spectrum: when the Auger and TA spectra are restricted to the commonly seen declinations, their cutoff energies are in a good agreement.

We have taken further steps to verify that the declination dependence of the TA SD spectrum is not an instrumental effect. In addition to the tests already performed in [13], we have re-examined the systematic uncertainties of our standard SD reconstruction by checking the linearity of the SD energy reconstruction with respect to the hadronic models as well as the FD in Section 5.1, and by cross-checking the results of our standard SD reconstruction with the Constant Intensity Cut method in Sections 5.2 and 5.3.

For a more detailed discussion on the recent Auger and TA spectrum comparisons, and the systematic uncertainties of the two experiments, an interested reader should consult the UHECR 2018 TA-Auger energy spectrum working group report [21].

\section{Acknowledgements}

The Telescope Array experiment is supported by the Japan Society for the Promotion of Science(JSPS) 

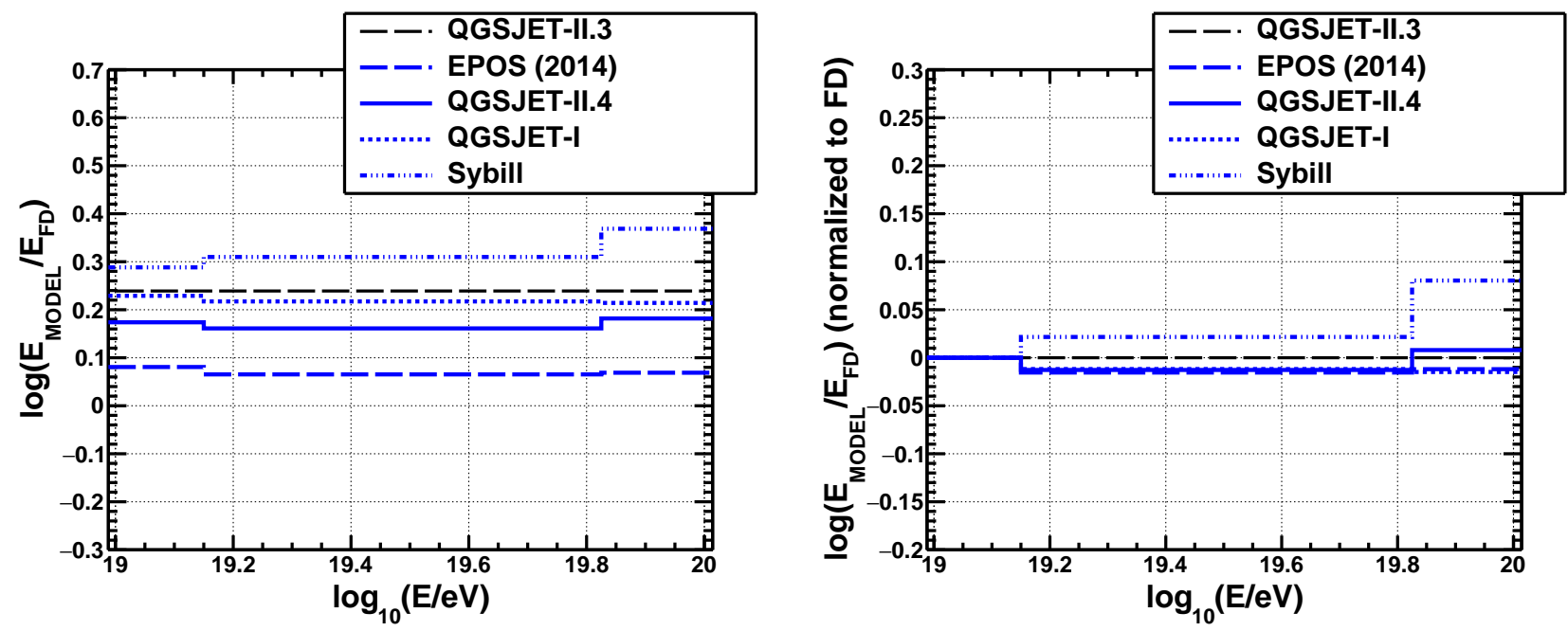

Figure 3. (LEFT): SD to FD calibration factors for a various hadronic interaction models (proton composition) as functions of true (Monte Carlo) energy. Same SD to FD calibration factors after dividing out the overall constants. In the extreme case of Sybill, the energy non-linearity is within $10 \%$ per decade of energy.

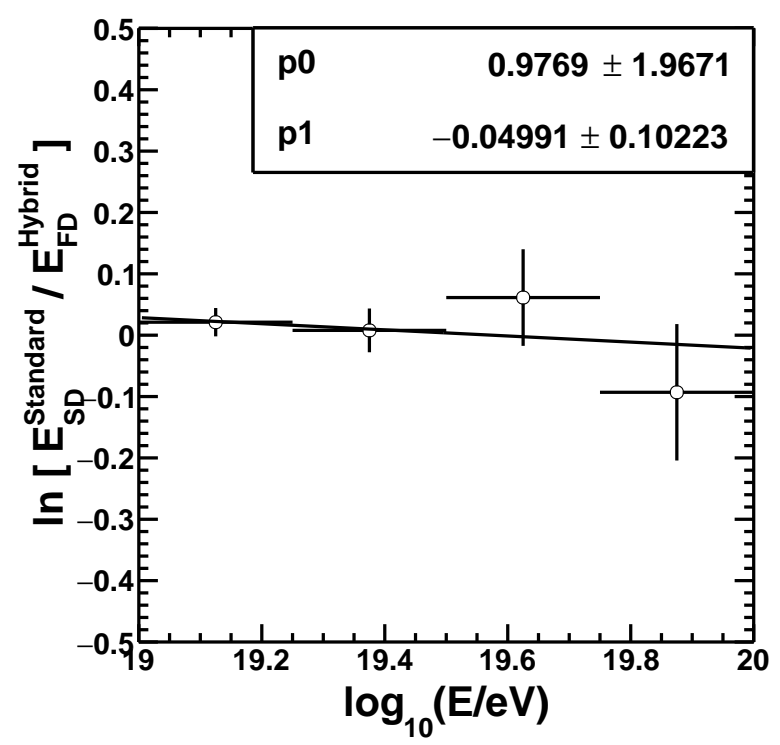

Figure 4. Natural log of the ratio of the calibrated surface detector energy to the fluorescence detector energy, plotted versus energy. A fit to a straight line gives a slope of -0.05 that is within its fitting uncertainty of 0.10 , indicating that there is no non-linearity of the SD energy with respect to the FD.

through Grants-in-Aid for Priority Area 431, for Specially Promoted Research JP21000002, for Scientific Research (S) JP19104006, for Specially Promoted Research JP15H05693, for Scientific Research (S) JP15H05741, for Science Research (A) JP18H03705 and for Young Scientists (A) JPH26707011; by the joint research program of the Institute for Cosmic Ray Research (ICRR), The University of Tokyo; by the U.S. National Science Foundation awards PHY-0601915, PHY-1404495, PHY-1404502, and PHY-1607727; by the National Research Foundation of Korea (2016R1A2B4014967, 2016R1A5A1013277, 2017K1A4A3015188, 2017R1A2A1A05071429); by the Russian Academy of Sciences, RFBR grant 16-02-00962a (INR), IISN project No. 4.4502.13, and Belgian Science Policy under IUAP VII/37 (ULB). The foundations of Dr. Ezekiel R. and Edna Wattis Dumke, Willard L. Eccles, and George S. and Dolores Doré Eccles all helped with generous donations. The State of Utah supported the project through its Economic Development Board, and the University of Utah through the Office of the Vice President for Research. The experimental site became available through the cooperation of the Utah School and Institutional Trust Lands Administration (SITLA), U.S. Bureau of Land Management (BLM), and the U.S. Air Force. We appreciate the assistance of the State of Utah and Fillmore offices of the BLM in crafting the Plan of Development for the site. Patrick Shea assisted the collaboration with valuable advice on a variety of topics. The people and the officials of Millard County, Utah have been a source of steadfast and warm support for our work which we greatly appreciate. We are indebted to the Millard County Road Department for their efforts to maintain and clear the roads which get us to our sites. We gratefully acknowledge the contribution from the technical staffs of our home institutions. An allocation of computer time from the Center for High Performance Computing at the University of Utah is gratefully acknowledged. 

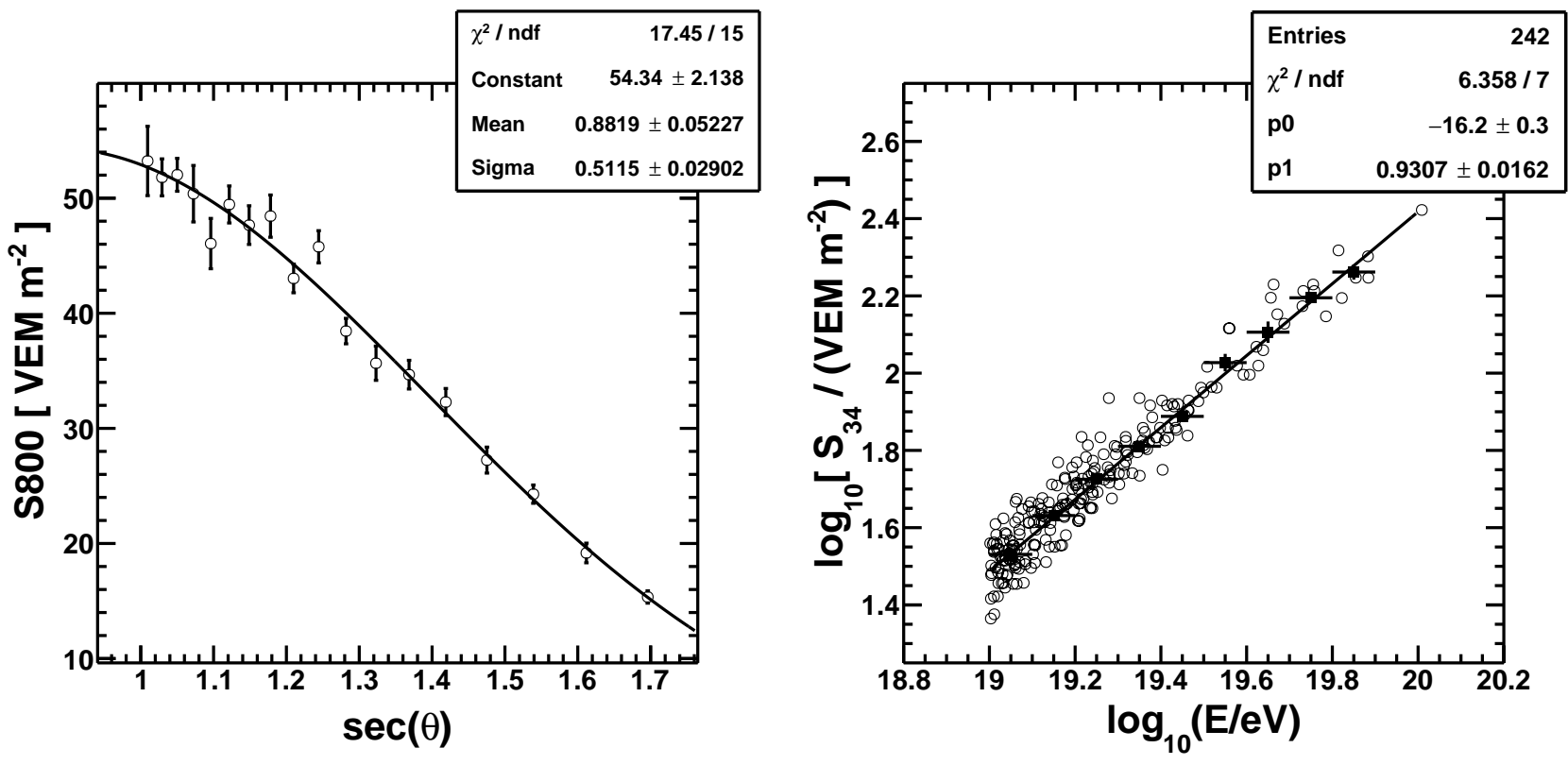

Figure 5. (LEFT): Minimum cuts on S800 that maintain a constant number of events in the equal-intensity angular bins for the TA SD data, plotted versus secant of the event zenith angle $(\theta)$. Solid line represents a fit to a Gaussian. Since the mean zenith angle of this data set is $34^{\circ}$, we choose to normalize the Constant Intensity Cut Curve (CIC) so that it is unity at $\sec \left(34^{\circ}\right)$. We define $S_{34}=\mathrm{S} 800 / \mathrm{CIC}(\sec (\theta))$ to be the estimator of the shower energy $(E)$. Physically, $S_{34}$ represents the S800 of a shower of the same energy $E$ but arriving at a zenith angle of $34^{\circ}$ instead of $\theta$. (RIGHT): Logarithm of the energy estimator $S_{34}$ plotted versus the logarithm of the energy for well reconstructed events that have been seen by both SD and FD. Open circles show the individual hybrid events, solid circles show the mean values of $\log _{10}\left(S_{34}\right)$ in the $\log _{10}(E)$ bins, and the solid line represents the linear fit.
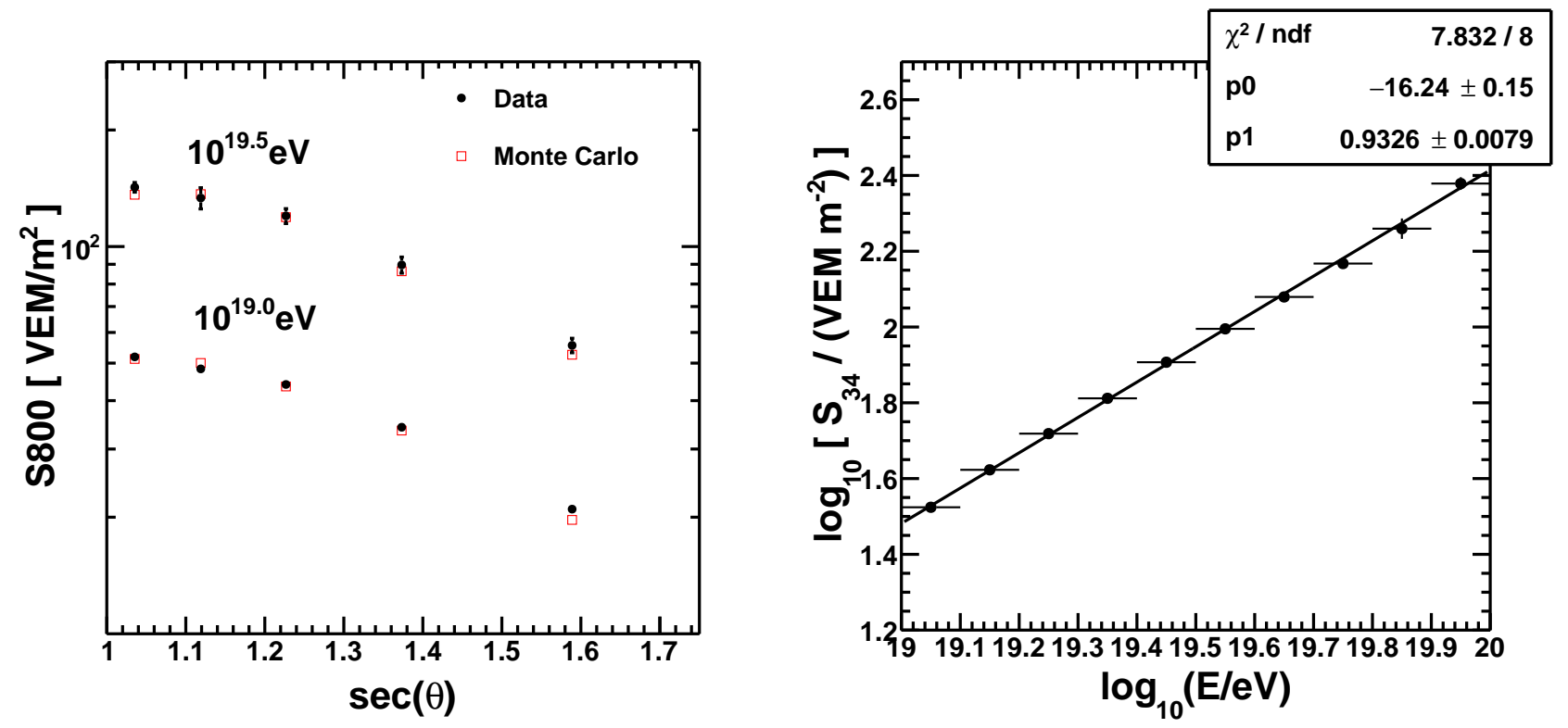

Figure 6. Left: The CIC attenuation comparisons between the data (black solid points) and the Monte Carlo (red open squares), for two energy thresholds, $10^{19.0}$ and $10^{19.5} \mathrm{eV}$. Right: The energy estimator $\log _{10}\left(S_{34}\right)$ plotted versus the logarithm of the true energy for the Monte Carlo events. Solid line shows the linear fit. 

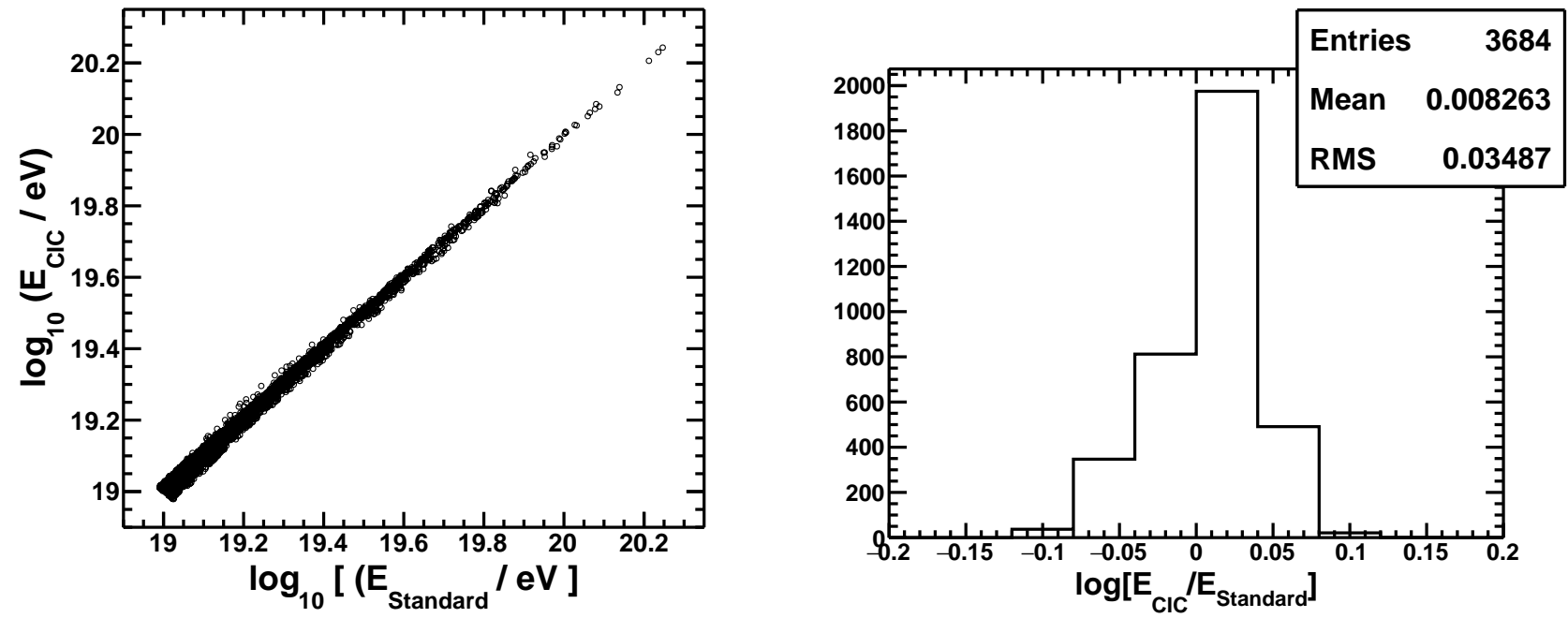

Figure 7. Event by event comparison of the TA SD standard and CIC reconstruction methods. Left panel shows the scatter plot and the right panel shows the ratio of the energies calculated by the standard and CIC methods.

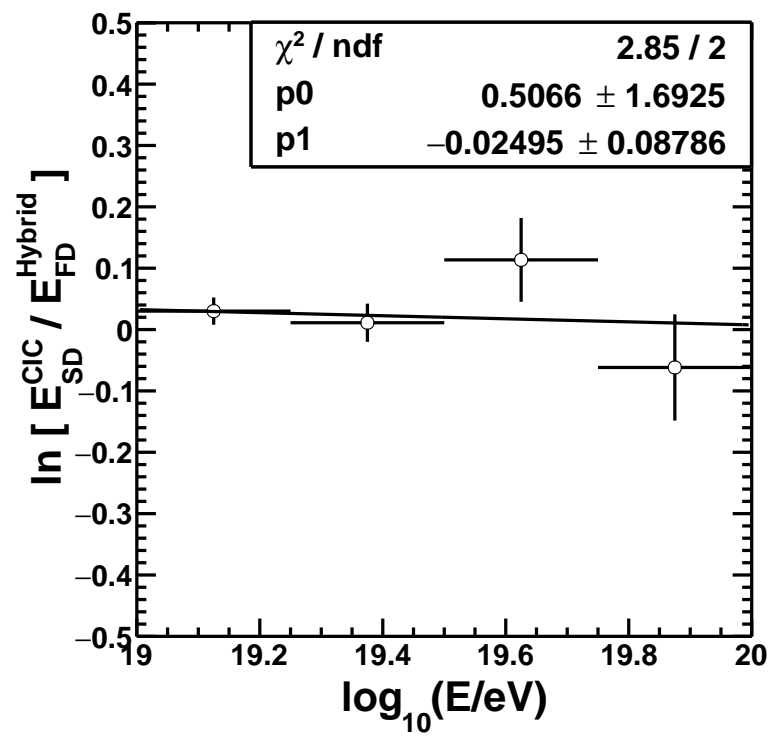

Figure 8. Natural log of the ratio of the TA SD energy estimated using the CIC method to the TA FD energy, plotted versus energy. A fit to a straight line gives a slope of -0.02 , which is within its fitting uncertainty of 0.09 , indicating that there is no non-linearity of the SD CIC energy with respect to the FD. 

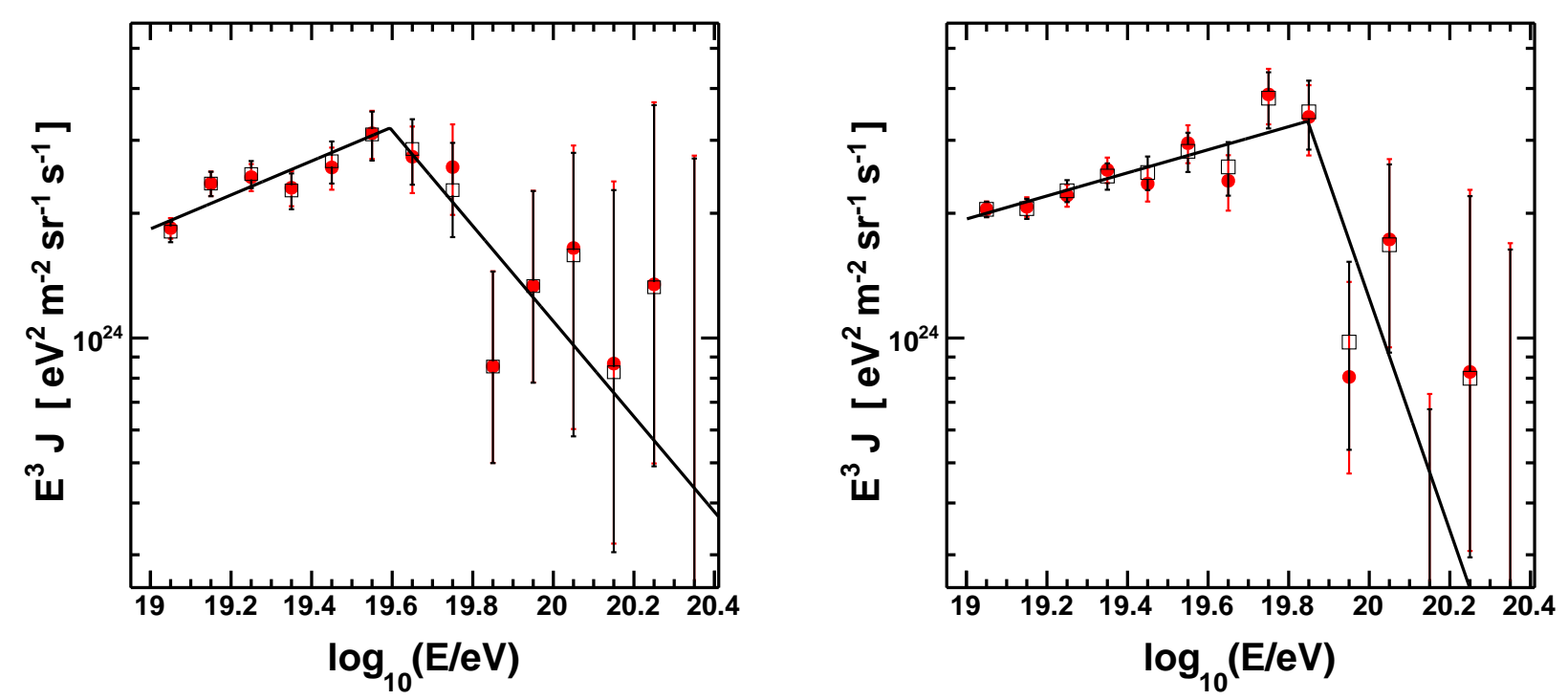

Figure 9. The TA SD energy spectra calculated using the CIC method, shown as red filled circles, and the standard TA method, shown as the open squares. The left panel compares the results for the lower declination band, $-15.7^{\circ}<\delta<24.8^{\circ}$, and the right panel compares the results for the higher declination band, $24.8^{\circ}<\delta<90^{\circ}$.

\section{References}

[1] J. Hersil, I. Escobar, D. Scott, G. Clark and S. Olbert, Phys. Rev. Lett. 6, 22 (1961)

[2] T. AbuZayyad, C.C. Jui, et. al UHECR 2018 conference

[3] R. U. Abbasi et al. [Telescope Array Collaboration], Astrophys. J. 865 (2018) no.1, 74 [arXiv:1803.01288 [astro-ph.HE]].

[4] T. Abu-Zayyad et al., arXiv:1803.07052 [astroph.HE].

[5] T. Abu-Zayyad et al. [Telescope Array Collaboration], Nucl. Instrum. Meth. A 689 (2012) 87 [arXiv:1201.4964 [astro-ph.IM]].

[6] T. Abu-Zayyad et al. [Telescope Array Collaboration], Nucl. Instrum. Meth. A 609 (2009) 227

[7] T. Abu-Zayyad et al. [Telescope Array Collaboration], Astropart. Phys. 39-40 (2012) 109 [arXiv:1202.5141 [astro-ph.IM]].

[8] W. D. Apel et al., Astropart. Phys. 36 (2012) 183

[9] F. Fenu [Pierre Auger Collaboration], PoS ICRC 2017 (2018) 486

[10] Y. Tsunesada, T. AbuZayyad, D. Ivanov, G. Thomson, T. Fujii and D. Ikeda, PoS ICRC 2017 (2018) 535

[11] T. AbuZayyad et al., Proceedings of the 2016 Conference on Ultrahigh Energy Cosmic Rays, Kyoto (Japan).
[12] D. Ivanov [Telescope-Array and Pierre Auger Collaborations], PoS ICRC 2017 (2018) 498

[13] D. Ivanov [Telescope Array Collaboration], PoS ICRC 2017 (2018) 496

[14] T. Abu-Zayyad et al. [Telescope Array Collaboration], Astrophys. J. 768 (2013) L1 [arXiv:1205.5067 [astro-ph.HE]]

[15] D. Ivanov, Energy Spectrum Measured by the Telescope Array Surface Detector, Ph.D. thesis, RutgersThe State University of New Jersey, Department of Physics and Astronomy, Piscataway, New Jersey, USA (October 2012).

[16] K. Shinozaki et al. [AGASA Collaboration], Nucl. Phys. Proc. Suppl. 136 (2004) 18

[17] J. Knapp and D. Heck, Nachr. Forsch. zentr. Karlsruhe 30 (1998) 27.

[18] S. Ostapchenko, Nucl. Phys. Proc. Suppl. 151 (2006) 143 [hep-ph/0412332].

[19] M. Kobal, Astropart. Phys. 15, (2001) 259

[20] B. T. Stokes, R. Cady, D. Ivanov, J. N. Matthews and G. B. Thomson, Astropart. Phys. 35 (2012) 759 [arXiv:1104.3182 [astro-ph.IM]].

[21] T. AbuZayyad et al., Proceedings of the 2018 Conference on Ultrahigh Energy Cosmic Rays, Paris (France). 